Books, videos, CD-ROMs, DVDs and any other relevant items submitted for a review in the $B D J$ should be addressed to: Kate Maynard, Assistant Editor, British Dental Journal, NPG, 4-6 Crinan Street, London N1 9XW
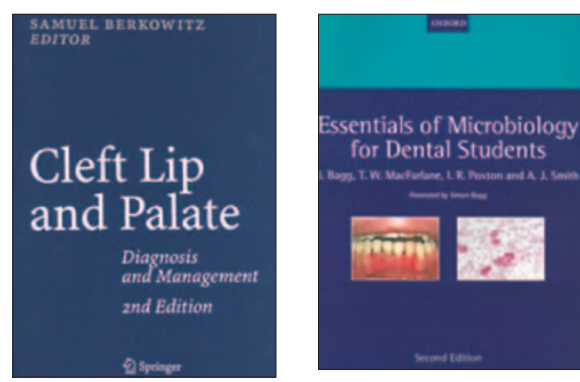

\section{Cleft lip and palate: Diagnosis and management}

\author{
S. Berkowitz (Ed) \\ Germany: Springer \\ price $€ 268.50$, pp 800 \\ ISBN 3540234098
}

This extremely comprehensive book has been edited by a world leader in the field of orofacial clefting. Through the text it is clear that Dr Berkowitz has achieved his goal of bringing together the contemporary concepts of diagnosis and management for patients with clefts. Although few will read this book coverto-cover, most readers will dip into one or two individual areas at a time. Dr Berkowitz has facilitated this by breaking the text into readable sections dealing with each issue. Students and experienced clinicians will find it easy to find the information they want quickly.

Although this is the second edition of a book first published in 1996, there have been numerous updates by the contributors of the original text commensurate with the fast changing pace of clinical research in the field of cleft lip and palate.

The book contains everything that most clinicians and researchers would want within a book about orofacial clefts: embryology, cleft typology, facial growth, surgery, presurgical orthopaedics/ comprehensive orthodontic treatment, bone grafting, speech and psychology. In the main, the text is supported by appropriate evidence. The results of some important studies are however underrepresented. I mention this not to criticise but to point out that each chapter contains opinions that some may disagree with. Space is allocated to a section on 'The future', detailing current clinical and molecular research and the potential promise of global collaborative research. This is a most worthwhile section, however within a few years it will become outdated and a third edition will inevitably be required because of scientific advances.
There is only one real criticism I would make. There is significant shine-through from the text and figures on the reverse side of some pages, reducing the quality of many of the excellent figures. This has no doubt arisen from the use of thinner paper in order to keep all 799 pages within one volume. Despite this, the text is illustrated with a vast array of figures, which for those embarking on a career working with patients with cleft lip and palate are extremely useful.

Would I buy this book? Not at $£ 268.50$, but I will certainly be borrowing it from my hospital library. It is an excellent resource for those involved in the multidisciplinary care of patients with clefts of the lip and palate as well as undergraduate and postgraduate students who merely wish to find out a little more information.

G. Mclntyre

\section{Essentials of}

\section{microbiology for dental students}

J. Bagg, T. W. MacFarlane, I. R. Poxton, A. J. Smith

UK: Oxford University Press

price €39.95, pp 317

ISBN 0198564899

Essentials of microbiology for dental students delivers exactly what it says in the title, succeeding in its stated aim to present microbiology in a clinical context that is relevant to the safe and effective practice of dentistry and providing a core reference text for dental undergraduates and postgraduates as well as for practising dentists.

This book has been produced with today's dental undergraduates firmly in mind, particularly from the perspective of the recommendation of the GDC document, The first five years, that basic science, including microbiology, should be closely integrated with clinical teaching and spread throughout the dental curriculum.

Not surprisingly, this second edition has retained the successful format of its predecessor which won the Dental Prize of the Medical Writers Group of the Society of Authors Medical Book Awards 2000. The text is written in a clear and concise style augmented by over 300 colour illustrations, photographs and tables. A 17 page glossary of scientific and clinical terms is a particularly useful feature as are the summary lists of key facts at the end of each chapter.

The book's 30 chapters are divided into three main sections dealing with: i) fundamental principles of microbiology, introducing infectious agents and host defence through to antimicrobial agents, disinfection and sterilisation and diagnostic microbiology; ii) infectious diseases relevant to dentistry presented in a system-based format; and iii) oral microbiology, providing an excellent introduction to the topic from which the student can progress to more detailed texts. The text has been updated since the previous edition in order to keep up with the recent developments in microbiology of which the dentist should be aware. New chapters have been written on fungi, human herpes viruses, infections of the central nervous system and the use of antimicrobial agents in dentistry. The emergence of infectious diseases such as severe acute respiratory syndrome (SARS) and prion diseases are also covered and accordingly the chapters on sterilisation, disinfection and infection control procedures in dentistry have been completely rewritten to reflect changes in decontamination protocols.

The topics are covered comprehensively, pitched of course at a level suited to dental rather than microbiology students, with suggested further reading at the end of each chapter.

As a convenor of a BDS Human Health and Disease course I have no hesitation in recommending this as a core textbook.

R. A. Whiley 